\title{
Gut Microbiota Shift in Obese Adolescents Born by Cesarean Section
}

\author{
Evgenia A. Novikova*; Natalia L. Belkova, PhD; Anna V. Pogodina, PhD, ScD; \\ Anastasia I. Romanitsa; Elizaveta S. Klimenko; Uliana M. Nemchenko, PhD; \\ Lyubov V. Rychkova, $\mathrm{PhD}, \mathrm{ScD}$, Corresponding Member of the RAS \\ Scientific Centre for Family Health and Human Reproduction Problems \\ Irkutsk, the Russian Federation
}

\begin{abstract}
Background: It is known that in the early postnatal period a variety of factors affect the gut microbiota (GM) composition, including delivery mode. The effect of delivery mode on the human GM in the late postnatal period remains unexplored. A shift of GM composition due to delivery mode may contribute to the development of obesity in adulthood.

Methods and Results: The study included six adolescents aged between 11 and 17 years treated and examined at the Clinic of the Scientific Center for Family Health and Human Reproduction (Irkutsk, Russia) in 2016. Stool samples were collected following the standard operating procedures according to the International Human Microbiome Standards. Metasequencing of V3-V4 variable regions of the 16S rRNA gene was performed by the Novogene Company (China) on the Illumina platform. Bioinformatic analysis was done by the bri-shur.com services. Sequencing reads were presented as normalized values.

In general, the GM composition of obese adolescents born by cesarean section was characterized by composition heterogeneity within the Bacteroidetes phylum and the dominance of certain phylotypes as signs of dysbiosis for each adolescent. We detected an increased abundance of phyla Bacteroides and Proteobacteria, and an absence of Tenericutes in obese adolescents born by Caesarean section. On the level of genera, the prevalence of Bacteroides and Bacteroides S24-7 phylotypes, and the absence of the RF39 phylotype, led to the GM shift associated with a cesarean section or obesity.

Conclusion: Obese adolescents born by cesarean section delivery present the shift in GM composition.(International Journal of Biomedicine. 2020;10(4):424-429.)
\end{abstract}

Key Words: gut microbiota $\bullet$ dysbiosis $\bullet$ amplicon metasequencing $\bullet$ cesarean section $\bullet$ vaginal birth $\bullet$ obesity $\bullet$ adolescents

\section{Abbreviations}

ALT, alanine transaminase; BMI, body mass index; BW, body weight; FPG, fasting plasma glucose; GM, gut microbiota; HDL, high-density lipoprotein; LDL, low-density lipoprotein; SDS BMI, standard deviation score of BMI; TG, triglycerides; TC, total cholesterol.

\section{Introduction}

The microbiota of the gastrointestinal tract constitute the complex, self-regulating microbial ecosystem that contains trillions of microorganisms. Gut bacteria play a fundamental role in human health by promoting intestinal homeostasis. It is known that a large number of factors affect the composition of

*Corresponding author: Evgenia A. Novikova, MD. Scientific Centre for Family Health and Human Reproduction Problems, Irkutsk, the Russian Federation.E-mail:europe411@mail.ru a child's gut microbiota (GM), such as the mode of delivery, ${ }^{(1-4)}$ the type of breastfeeding, ${ }^{(5,6)}$ and antibiotic therapy. ${ }^{(7)}$ During the perinatal period immediately after vaginal delivery, the infant intestine is colonized by maternal bacteria belonging to Bacteroides, Bifidobacterium, Parabacteroides, Escherichia, Shigella ${ }^{\left({ }^{(8)}\right.}$ Lactobacillus, and Prevotella ${ }^{(9)}$ This colonization process is a massive influx of antigens, and it provides a physiological adaptation for infants. cesarean delivery breaks the normal colonization of the infant gut by preventing exposure to maternal microbes. The intestine of infants delivered by cesarean delivery is colonized by bacteria typical 
for the skin, such as Staphylococcus, Corynebacterium, and Propionibacterium, ${ }^{(10)}$ and has an increase of Clostridium difficile. It also has a slowed growth of Bacteroides, Bifidobacterium, and Escherichia coli, compared to vaginally born babies. ${ }^{(10,11)}$

Multiple studies suggest that cesarean delivery has a postnatal impact on children's GM. Some of these studies show an imbalance in GM composition or dysbiosis among infants between two and six weeks of age,,$^{(1,2)}$ whereas other data point to microbiota heterogeneity persisting up to a year, $(3,4)$ or extending up to seven years. ${ }^{(12)}$ The influence of delivery mode on the ${ }^{\mathrm{GM}}$ composition in the late postnatal period has been little studied, especially in the teenage period, as a critical period for health.

Moreover, in the last decades, GM was acknowledged as one of the key pathogenic factors affecting many components of the metabolic syndrome and obesity. Dysbiosis may contribute to a predisposition for diseases, including obesity formation. ${ }^{(13-15)}$ A deep understanding of the factors provoking GM dysbiosis in lifelong health is needed.

In this study, we checked the GM composition in adolescents born by cesarean section and those born by vaginal delivery with an assessment of how that composition contributes to the development of obesity.

\section{Materials and Methods}

The study was approved by the Ethics Committee of the Scientific Center for Family Health and Human Reproduction Problems. Written informed consent was obtained from each research participant (or the participant's parent/guardian).

Exclusion criteria were intake of prebiotics or probiotics in the previous six months, antibiotic therapy in the previous three months, chronic inflammatory bowel diseases in the past, severe somatic pathologies, and intestinal infections in the last six months in anamnesis.

The study included six adolescents aged between 11 and 17 years with normal weight (SDS BMI $<1.0$ ) or obesity (SDS BMI $\geq 2.0)^{(16)}$ treated and examined at the Clinic of the Scientific Center for Family Health and Human Reproduction (Irkutsk, Russia) in 2016. Three adolescents were born by cesarean delivery: One male adolescent was within the normal weight range (D38-m), the other two participants, male and female, had obesity (D10-m and D16-f). For comparison, three adolescents who were born vaginally were also included. Matched pairs of adolescents had the same gender, and were comparable for breastfeeding duration and age, and were born by a vaginal delivery. One male adolescent was within the normal weight range (D44-m); the other two, a male and a female, had obesity-D39-m and D12-f, respectively. The clinical characteristics of participants are presented in Table 1.

The concentration of FPG, TC, TG, HDL, LDL, and serum ALT was measured using a Mindray Automatic Biochemistry Analyzer. The reference for LDL was less than 3.36. ${ }^{(17)}$ The reference for ALT was less than 25IU/1. ${ }^{(18)}$ The reference of atherogenic index was less than 3.0. ${ }^{(19)}$

Stool samples were collected following the standard operating procedures according to the International Human
Microbiome Standards. The quality of DNA was assessed in $1 \%$ agarose gel electrophoresis, DNA concentration was measured using the NanoDrop ${ }^{\mathrm{TM}}$ spectrophotometer (Thermo Scientific, USA). Metasequencing of V3-V4 variable regions of the 16S rRNA gene was performed by the Novogene Company (China) on the Illumina platform. Bioinformatic analysis was done by the bri-shur.com services. ${ }^{(2)}$ Sequencing reads were presented as normalized values.

Table 1.

\section{Clinical characteristics of participants}

\begin{tabular}{|c|c|c|c|c|c|c|}
\hline \multirow{3}{*}{ Variable } & \multicolumn{3}{|c|}{ Cesarean delivery } & \multicolumn{3}{|c|}{ Vaginal delivery } \\
\hline & \multicolumn{2}{|c|}{ Obesity } & \multirow{2}{*}{\begin{tabular}{|l}
$\begin{array}{c}\text { Normal } \\
\text { weight }\end{array}$ \\
D38-m \\
\end{tabular}} & \multicolumn{2}{|c|}{ Obesity } & \multirow{2}{*}{$\begin{array}{l}\text { Normal } \\
\text { weight }\end{array}$} \\
\hline & D10-m & D16-f & & D39-m & D12-f & \\
\hline Gender & male & female & male & male & female & male \\
\hline Age, years & 11 & 11 & 17 & 13 & 15 & 17 \\
\hline $\begin{array}{l}\text { Breastfeeding } \\
\text { duration }>3 \text { month }\end{array}$ & yes & yes & none & yes & yes & none \\
\hline BMI, $\mathrm{kg} / \mathrm{m}^{2}$ & 30.4 & 33.0 & 21.8 & 29.1 & 37.4 & 22.0 \\
\hline SDS BMI & 3.3 & 3.4 & 0.1 & 2.6 & 3.2 & 0.3 \\
\hline $\mathrm{TC}, \mathrm{mmol} / \mathrm{L}$ & 4.98 & 4.97 & 4.99 & 4.46 & 4.98 & 3.00 \\
\hline TAG, $\mathrm{mmol} / \mathrm{L}$ & 0.98 & 0.89 & 1.14 & 1.24 & 0.85 & 0.45 \\
\hline $\mathrm{HDL}, \mathrm{mmol} / \mathrm{L}$ & 1.16 & 1.52 & 1.50 & 1.00 & 1.72 & NA \\
\hline $\mathrm{LDL}, \mathrm{mmol} / \mathrm{L}$ & $3.37 *$ & 3.04 & 2.97 & 2.90 & 2.87 & NA \\
\hline Atherogenic index & $3.3 *$ & 2.3 & 2.3 & $3.5^{*}$ & 1.9 & NA \\
\hline FPG, mmol/L & 4.8 & 4.0 & 4.1 & 5.0 & 5.5 & 3.9 \\
\hline ALT, IU/L & $26.0 *$ & 24.8 & 13.8 & 23.4 & 12.6 & 27.4 \\
\hline
\end{tabular}

Note: * above the reference, $N A$ - not available data

\section{Results and Discussion}

\section{GM composition of adolescents at the phylum and class level}

We found changes in GM composition for adolescents, depending on BW or delivery mode. We have seen the shift of GM composition in obese adolescents born by cesarean section already at the high taxonomic level. Compared with obese adolescents who were delivered vaginally, those born by cesarean delivery had bacterial communities with a higher abundance of the phyla Bacteroidetes and Proteobacteria, and a lower abundance of Firmicutes and absence of Tenericutes (Table 2). Other phyla, such as Actinobacteria and Verrucomicrobia, were represented in a similar abundance for adolescents with different BW who had been delivered via a different mode.

At the level of classes and genera, the shift in GM composition was found to be more prominent. Obese adolescents born by cesarean section had an increased abundance of the Bacteroidia class (the Bacteroidetes phylum) and a decrease of Clostridia (the Firmicutes phylum). Figure 1 shows the top 25 most abundant genera of the individual gut microbiomes of adolescents born by vaginal (A) and cesarean (B) delivery. All three vaginally born adolescents, regardless of $\mathrm{BW}$, shared bacterial genera belonging to Clostridia, Bacteroidia, Gammaproteobacteria, Betaproteobacteria, and Deltaproteobacteria classes. They 
formed the common bacterial core. The GM composition of adolescents born by cesarean section was different for each of them without the bacterial core. This fact illustrates how the mode of delivery is essential for GM formation.

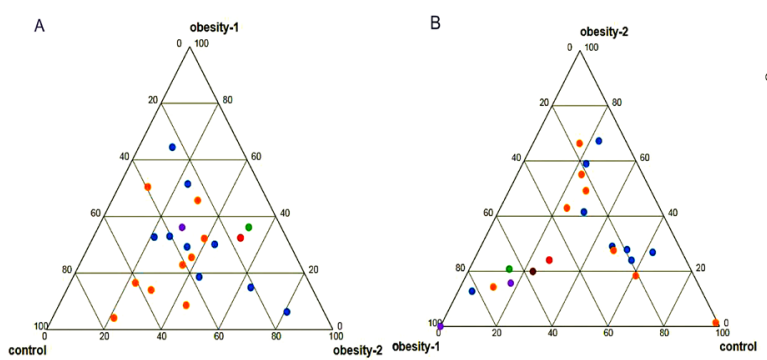

Fig. 1. The top 25 most abundant genera of the individual gut microbiomes of adolescents born by vaginal (A) and cesarean (B) delivery.

Note: obesity-1 and obesity-2 - obese adolescents, control normal-weight adolescents
Bacteroidetes, Firmicutes, and Proteobacteria are the three most abundant bacterial phyla of adult GM. Their ratio is a very important sign of healthy GM composition. This finding does not concur with previous metagenomic studies that demonstrated a lower amount of Bacteroidetes and a higher amount of Firmicutes for obese people than for lean controls. ${ }^{(21)}$ Such contradictions can be explained by including in our study the delivery mode as the factor influencing GM composition. Regarding Proteobacteria, especially the Gammaproteobacteria class, on the one hand, its members such as enterococci and E. coli are part of the healthy GM composition; (22) on the other hand, many opportunistic microorganisms belong to this class. ${ }^{(23)}$ Therefore, very often, an increased abundance of Gammaproteobacteria was found to be associated with gut dysbiosis.

Other phyla are presented in GM composition in a considerably smaller amount, but nonetheless play an important role in the GM metabolism. Actinobacteria and Verrucomicrobia belong to the healthy GM composition, and they have

Table 2.

Abundance of dominant phyla, classes and their ratios depending on delivery mode and body weight

\begin{tabular}{|c|c|c|c|c|c|c|}
\hline \multirow{3}{*}{ Taxonomic level } & \multicolumn{3}{|c|}{ Cesarean delivery } & \multicolumn{3}{|c|}{ Vaginal delivery } \\
\hline & \multicolumn{2}{|c|}{ Obesity } & \multirow{2}{*}{$\begin{array}{c}\text { Normal } \\
\text { weight }\end{array}$} & \multicolumn{2}{|c|}{ Obesity } & \multirow{2}{*}{$\begin{array}{c}\begin{array}{c}\text { Normal } \\
\text { weight }\end{array} \\
\text { D44-m }\end{array}$} \\
\hline & D10-m & D16-f & & D39-m & D12-f & \\
\hline \multicolumn{7}{|c|}{ Phylum } \\
\hline Bacteroidetes & 63.4 & 61.3 & 49.9 & 62.8 & 53.7 & 53.1 \\
\hline Firmicutes & 19.7 & 35.2 & 45.6 & 33.0 & 40.6 & 41.5 \\
\hline Proteobacteria & 13.9 & 2.5 & 2.8 & 3.3 & 4.2 & 2.3 \\
\hline Actinobacteria & 1.5 & 0.7 & 0.7 & 0.4 & 1.4 & 1.1 \\
\hline Verrucomicrobia & 0.3 & 0.3 & 0.2 & 0.4 & 0.1 & 0.2 \\
\hline Tenericutes & 0 & 0 & 0.8 & 0.1 & 0 & 1.7 \\
\hline \multicolumn{7}{|c|}{ Ratios for phyla } \\
\hline Bacteroidetes/Firmicutes & 3.2 & 1.7 & 1.1 & 1.9 & 1.3 & 1.3 \\
\hline Proteobacteria/Actinobacteria & 9.3 & 3.6 & 4.0 & 8.3 & 3.0 & 2.1 \\
\hline \multicolumn{7}{|c|}{ Class (phylum) } \\
\hline Bacteroidia (Bacteroidetes) & 63.4 & 61.3 & 49.9 & 62.8 & 53.7 & 53.1 \\
\hline Clostridia (Firmicutes) & 18.6 & 34.6 & 45.0 & 32.8 & 40.0 & 40.4 \\
\hline Bacilli (Firmicutes) & 1.1 & 0.1 & 0.1 & 0.1 & 0.3 & 0.4 \\
\hline Erysipelotrichi (Firmicutes) & 0.1 & 0.4 & 0.4 & 0.2 & 0.3 & 0.8 \\
\hline Gammaproteobacteria (Proteobacteria) & 11.1 & 1.2 & 1.4 & 1.4 & 2.3 & 1.0 \\
\hline Betaproteobacteria (Proteobacteria) & 1.6 & 0.8 & 1.0 & 0.9 & 1.2 & 0.3 \\
\hline Deltaproteobacteria (Proteobacteria) & 1.0 & 0.5 & 0.5 & 1.0 & 0.7 & 0.9 \\
\hline Actinobacteria (Actinobacteria) & 1.1 & 0.4 & 0.3 & 0.2 & 1.0 & 0.4 \\
\hline Coriobacteriia (Actinobacteria) & 0.4 & 0.4 & 0.4 & 0.2 & 0.4 & 0.7 \\
\hline \multicolumn{7}{|c|}{ Ratios for classes } \\
\hline Bacteroidia/Clostridia & 3.4 & 1.8 & 1.1 & 1.9 & 1.3 & 1.3 \\
\hline $\begin{array}{l}\text { Gammaproteobacteria/ } \\
\text { Actinobacteria }\end{array}$ & 10.1 & 3.0 & 4.7 & 7.0 & 2.3 & 2.5 \\
\hline Clostridia/Bacilli & 16.9 & 346.0 & 450.0 & 328.0 & 133.3 & 101.0 \\
\hline Clostridia/Erysipelotrichi & 186.0 & 86.5 & 112.5 & 164.0 & 133.3 & 50.5 \\
\hline Bacteroidia/Bacilli & 57.6 & 613.0 & 499.0 & 628.0 & 179.0 & 132.8 \\
\hline Bacteroidia/Erysipelotrichi & 634.0 & 153.3 & 124.8 & 314.0 & 179.0 & 66.4 \\
\hline Betaproteobacteria/ Actinobacteria & 1.5 & 2.0 & 3.3 & 4.5 & 1.2 & 0.8 \\
\hline Deltaproteobacteria/ Actinobacteria & 0.9 & 1.3 & 1.7 & 5.0 & 0.7 & 2.3 \\
\hline
\end{tabular}

Note: $m$-male, $f$-female 
Bifidobacterium and Akkermansia as their members. ${ }^{(24,25)} \mathrm{We}$ did not observe differences for these phyla between adolescents born vaginally and those born by cesarean section, which probably can be explained by the fact that these phyla are balanced by others. However, our study detected no bacteria belonging to the Tenericutes phylum for obese adolescents born by cesarean section. Tenericutes play an essential role in the host metabolism as intracellular microorganisms and may change intracellular metabolism. ${ }^{(26)}$ Contradictions between the results of current findings and those of previous studies can be explained by including the delivery mode as a factor influencing GM composition.

\section{GM composition of adolescents at the phylotype level}

Taxonomic-level differences in GM composition were found for several phylotypes. Table 3 shows the most abundant 20 bacterial phylotypes in the gut microbiome of adolescents with different delivery mode and BW. In general,

\section{Table 3.}

The top 20 most abundant bacterial phylotypes of adolescents with different delivery mode and body weight

\begin{tabular}{|c|c|c|c|c|c|c|}
\hline \multirow{3}{*}{ Phylotype } & \multicolumn{3}{|c|}{ Cesarean delivery } & \multicolumn{3}{|c|}{ Vaginal delivery } \\
\hline & \multicolumn{2}{|c|}{ Obesity } & NW & \multicolumn{2}{|c|}{ Obesity } & NW \\
\hline & \multicolumn{6}{|c|}{ D10-m D16-f L } \\
\hline \multicolumn{7}{|c|}{ Bacteroidetes } \\
\hline Prevotella & 47.4 & 9.2 & 7.6 & 40.1 & 26.3 & 21.4 \\
\hline Bacteroides & 11.5 & 44.8 & 11.3 & 14.3 & 17.3 & 12.8 \\
\hline PL_6_Bacteroides S24-7* & 0.2 & 0.3 & 23.7 & 1.7 & 2.4 & 6.4 \\
\hline PL_5_Rikenellaceae & 1.3 & 1.4 & 2.5 & 0.9 & 1.8 & 3.5 \\
\hline PL_4_Bacteroidales & 0.1 & 0.1 & 0.1 & 0.1 & 0.6 & 2.2 \\
\hline PL_6_[Prevotella] & 0.5 & 0.7 & 0.4 & 2.3 & 0.5 & 1.8 \\
\hline Parabacteroides & 1.1 & 2.3 & 1.3 & 1.2 & 1.8 & 1.8 \\
\hline PL_5_[Barnesiellaceae] & 0.6 & 1.6 & 0.7 & 0.8 & 1.3 & 1.5 \\
\hline \multicolumn{7}{|c|}{ Firmicutes } \\
\hline PL_5_Ruminococcaceae & 5.3 & 6.5 & 15.3 & 11 & 8.9 & 13.6 \\
\hline PL_5_Lachnospiraceae & 1.4 & 9.6 & 3.3 & 3.3 & 7.8 & 6.6 \\
\hline PL_4_Clostridiales & 2.1 & 5.4 & 12.5 & 4.6 & 5.5 & 5.8 \\
\hline Dialister & 1.1 & 3.5 & 1.4 & 2.9 & 1.9 & 4.1 \\
\hline Faecalibacterium & 1.2 & 1.9 & 1.4 & 1.4 & 6 & 2 \\
\hline Ruminococcus & 1.6 & 2.3 & 4.4 & 2.1 & 3.1 & 1.9 \\
\hline Oscillospira & 1.2 & 1.5 & 2.3 & 2.2 & 1 & 1.1 \\
\hline Megasphaera & 2.3 & 0.4 & 0.1 & 2.2 & 0.4 & 0.8 \\
\hline \multicolumn{7}{|c|}{ Proteobacteria } \\
\hline PL_5_Enterobacteriaceae & 4.8 & 11 & 1.3 & 1.3 & 2.1 & 0.7 \\
\hline Sutterella & 1.5 & 0.8 & 0.8 & 0.8 & 1.2 & 0.2 \\
\hline PL_5_Xanthomonadaceae & 5.6 & 0 & 0 & 0 & 0 & 0 \\
\hline \multicolumn{7}{|c|}{ Tenericutes } \\
\hline PL_6_RF39 & 0 & 0 & 0.7 & 0.1 & 0 & 1.6 \\
\hline
\end{tabular}

Note: $N W$-Normal weight; $m$-male, $f$-female, * PL-phylotype level-the level of identification, 4 - the order, 5 - the family, 6 - the genus the GM composition of obese adolescents born by cesarean section was characterized by composition heterogeneity within the Bacteroidetes phylum and the dominance of certain phylotypes as signs of dysbiosis for each adolescent.

As others have reported, we observed that vaginally born adolescents had a slightly higher representation of the Bacteroides genus than the cesarean born. Thus, Madan et al. showed vaginal delivery is associated with an increased number of Bacteroides $(P=0.0007, \mathrm{q}=0.02)$ for infants. ${ }^{(2)}$ In addition, the obese female adolescent born by cesarean section has twice the increase of Bacteroides as adolescents born by vaginal delivery; this is in contradiction to some research reports showing an absence of Bacteroides for infants born by cesarean delivery. ${ }^{(3)}$ Present controversies may be explained by a difference in age among participants. GM of adolescents must be more stable and adult-like than it is during the early childhood period. However, according to our results those who were born by cesarean delivery still present the shift in bacterial composition.

Another huge inequality was found for the phylotype Bacteroides S24-7, which has structural similarities to Bacteroides genus. This phylotype was considerably more represented in GM of normal weight adolescents than Bacteroides itself. In contrast, the obese adolescent compensates for the decrease in Bacteroides S24-7 by an increase in Lachnospiraceae phylotype (the Firmicutes phylum). This evidence is in agreement with the data suggesting the participation of Firmicutes in the deposition of fats.

The GM heterogeneity is characterized by an appearance of new members of a bacterial community. Against this background some community members may get lost. For example, GM of obese adolescents born by cesarean section was characterized by an absence of the RF39 phylotype (the Tenericutes phylum).

In contrast, samples of vaginally born adolescents were homogeneous in the Bacteroidetes phylum, which indicates a stable healthy GM state. Therefore, the normal weight adolescents born by vaginal delivery showed increased values for all the most abundant phylotypes of the Bacteroidetes phylum equally (Table 3 ), which confirms the literature data that shows an increased abundance of the members of phylum Bacteroidetes in normal weight children. ${ }^{(21)}$

Therefore, the increased abundance of separate phylotypes among the top 20 most abundant bacterial phylotypes may indicate that GM participates in the formation of obesity among adolescents born by cesarean section.

Features of the metabolic pathways in GM adolescents

The association of microbial and host metabolism is well known; ${ }^{(27)}$ thus, an imbalance in the gut microbiome may contribute to metabolic changes in the host. We made a model of the metabolic pathways of adolescent gut microbiomes based on a dataset of paired interactions (according to the present/ absent principle) among the 25 most represented phylotypes (Figure 2). The metabolic pathways of gut microbiomes cross each other for all three adolescents born vaginally. However, the metabolic pathways of gut bacteria of three adolescents born by cesarean section are completely autonomous, unique, 
and do not have common contact points. Later we analyzed biochemical parameters of obese adolescents born by cesarean section and found some values above the reference numbers (Table 1). LDL of the male adolescent with obesity was 3.37 $\mathrm{mmol} / \mathrm{L}$, and ALT was $260 \mathrm{IU} / \mathrm{L}$. In addition, the atherogenic index of male adolescents with obesity was increased regardless of delivery mode. It is likely that the metabolism of adolescents born by cesarean section can be obesogenic due to altered GM composition, but the concrete metabolic pathways underlying these observations remain unknown.
A

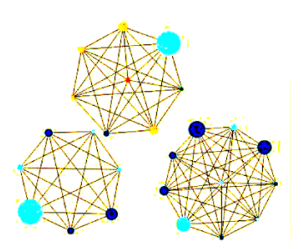

B

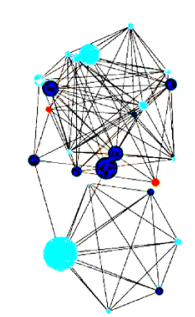

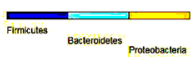

Fig. 2. Visualization of the possible metabolic pathways of the 25 most represented phylotypes of gut microbiomes of adolescents born by cesarean (A) and vaginal $(B)$ delivery. Note: two cases with obesity stick together, the third one with normal weight lies apart from them

In conclusion, understanding the factors provoking GM dysbiosis in adolescents is critical for determining at an early stage of life any predisposition to certain illnesses, namely obesity. To this end, using the metagenome analysis, we showed a shift in the GM composition of obese adolescents born by cesarean section both at the level of large taxa and at the level of lower taxonomic units. We described the shift for adolescents, as opposed to numerous studies showing changes in the microbiome of young children. This fact makes our study important and helpful for understanding the mechanisms of keeping GM dysbiosis at later stage of life.

\section{Competing Interests}

The authors declare that they have no competing interests.

\section{References}

1. Kim G, Bae J, Kim MJ, Kwon H, Park G, Kim S-J et al. Delayed Establishment of Gut Microbiota in Infants Delivered by Cesarean Section. Front Microbiol. 2020 Sep 11;11:2099. doi: $10.3389 /$ fmicb.2020.02099.

2. Madan JC, Hoen AG, Lundgren SN, Farzan SF, Cottingham KL, Morrison HG, et al. Association of Cesarean Delivery and Formula Supplementation With the Intestinal Microbiome of 6-Week-Old Infants. JAMA Pediatr. 2016;170(3):212-9. doi: 10.1001/jamapediatrics.2015.3732.

3. Azad MB, Konya T, Maughan H, Guttman DS, Field CJ,
Chari RS, et al.; CHILD Study Investigators. Gut microbiota of healthy Canadian infants: profiles by mode of delivery and infant diet at 4 months. CMAJ. 2013;185(5):385-94. doi: 10.1503/cmaj.121189.

4. Reyman M, van Houten MA, van Baarle D, Bosch AATM, Man WH, Chu MLJN, wt al. Impact of delivery modeassociated gut microbiota dynamics on health in the first year of life. Nat Commun. 2019 Nov 1;10(1):4997. doi: 10.1038/ s41467-019-13014-7. Erratum in: Nat Commun. 2019 Nov 25;10(1):5352.

5. Belkova NL, Nemchenko UM, Pogodina AV, Feranchuk SI, Romanitsa AI, Novikova EA, Rychkova LV. Composition and Structure of Gut Microbiome in Adolescents with Obesity and Different Breastfeeding Duration. Bull Exp Biol Med. 2019 Oct;167(6):759-762. doi: 10.1007/s10517-019-04617-7. 6. Pannaraj PS, Li F, Cerini C, Bender JM, Yang S, Rollie A, Adisetiyo H, Zabih S, Lincez PJ, Bittinger K, Bailey A, Bushman FD, Sleasman JW, Aldrovandi GM. Association Between Breast Milk Bacterial Communities and Establishment and Development of the Infant Gut Microbiome. JAMA Pediatr. 2017 Jul 1;171(7):647-654. doi: 10.1001/jamapediatrics.2017.0378.

7. Fjalstad JW, Esaiassen E, Juvet LK, van den Anker JN, Klingenberg C. Antibiotic therapy in neonates and impact on gut microbiota and antibiotic resistance development: a systematic review. J Antimicrob Chemother. 2018 Mar 1;73(3):569-580. doi: 10.1093/jac/dkx426.

8. Gosalbes MJ, Llop S, Vallès Y, Moya A, Ballester F, Francino MP. Meconium microbiota types dominated by lactic acid or enteric bacteria are differentially associated with maternal eczema and respiratory problems in infants. Clin Exp Allergy. 2013 Feb;43(2):198-211. doi: 10.1111/cea.12063.

9. Wang L, Gong Z, Huo J, Zhuo Q, Qin W, Yang Z, Wang J, Shen S. [Effects of nutrition package on intestinal flora of infants by high-throughput sequencing]. Wei Sheng Yan Jiu. 2020 Mar;49(2):233-237. Chinese. doi: 10.19813/j.cnki. weishengyanjiu.2020.02.011. [Article in Chinese].

10. Mueller NT, Bakacs E, Combellick J, Grigoryan Z, Dominguez-Bello MG. The infant microbiome development: mom matters. Trends Mol Med. 2015 Feb;21(2):109-17. doi: 10.1016/j.molmed.2014.12.002. E

11. Marques TM, Wall R, Ross RP, Fitzgerald GF, Ryan CA, Stanton C. Programming infant gut microbiota: influence of dietary and environmental factors. Curr Opin Biotechnol. 2010 Apr;21(2):149-56. doi: 10.1016/j.copbio.2010.03.020.

12. Nikolaeva IV, Tsaregorodtsev AD, Shaikhieva GS. [Formation of the intestinal microbiota of children and the factors that influence this process]. Rossiyskiy Vestnik Perinatologii i Pediatrii (Russian Bulletin of Perinatology and Pediatrics). 2018;63(3):13-8. doi: 10.21508/1027-4065-201863-3-13-18.p. [Article in Russian].

13. Huurre A, Kalliomäki M, Rautava S, Rinne M, Salminen $\mathrm{S}$, Isolauri E. Mode of delivery - effects on gut microbiota and humoral immunity. Neonatology. 2008;93(4):236-40. doi: 10.1159/000111102.

14. Miettinen R, Hermansson H, Merikukka M, Gissler M, IsolauriE. Mode of delivery--impact on risk of noncommunicable diseases. J Allergy Clin Immunol. 2015 Nov;136(5):1398-9. doi: 10.1016/j.jaci.2015.05.032.

15. Kalliomäki M, Collado MC, Salminen S, Isolauri E. Early differences in fecal microbiota composition in children may predict overweight. Am J Clin Nutr. 2008 Mar;87(3):534-8. doi: 10.1093/ajen/87.3.534. 
16. WHO. Obesity and overweight. Available at: https://www. who.int/news-room/fact-sheets/detail/obesity-and-overweight (accessed 2020 September 30).

17. Friedewald WT, Levy RI, Fredrickson DS. Estimation of the concentration of low-density lipoprotein cholesterol in plasma, without use of the preparative ultracentrifuge. Clin Chem. 1972 Jun;18(6):499-502.

18. Styne DM, Arslanian SA, Connor EL, Farooqi IS, Murad MH, Silverstein JH, Yanovski JA. Pediatric ObesityAssessment, Treatment, and Prevention: An Endocrine Society Clinical Practice Guideline. J Clin Endocrinol Metab. 2017 Mar 1;102(3):709-757. doi: 10.1210/jc.2016-2573.

19. Klimov AN. Causes and conditions of atherosclerosis development. In: Kositsky GI, editors. Preventive cardiology. Moscow: Meditsina; 1977:260-321.[In Russian].

20. Feranchuk S, Belkova N, Potapova U, Ochirov I, Kuzmin D, Belikov S. [Tools and a web server for data analysis and visualization in microbial ecology]. Community Ecology. 2019;20(2):230-237. [Article in Russian].

21. Ley RE, Turnbaugh PJ, Klein S, Gordon JI. Microbial ecology: human gut microbes associated with obesity. Nature. 2006 Dec 21;444(7122):1022-3. doi: 10.1038/4441022a.

22. Tenaillon O, Skurnik D, Picard B, Denamur E. The population genetics of commensal Escherichia coli. Nat Rev Microbiol. 2010 Mar;8(3):207-17. doi: 10.1038/nrmicro2298. 23. Brubaker RR. Physiology of Yersinia pestis. Adv Exp Med Biol. 2016;918:79-99. doi: 10.1007/978-94-024-0890-4 4.

24. Hidalgo-Cantabrana C, Delgado S, Ruiz L, Ruas-Madiedo P, Sánchez B, Margolles A. Bifidobacteria and Their HealthPromoting Effects. Microbiol Spectr. 2017 Jun;5(3). doi: 10.1128/microbiolspec.BAD-0010-2016.

25. Geerlings SY, Kostopoulos I, de Vos WM, Belzer C. Akkermansia muciniphila in the Human Gastrointestinal Tract: When, Where, and How? Microorganisms. 2018 Jul 23;6(3):75. doi: 10.3390/microorganisms6030075.

26. Trachtenberg S. Mollicutes. Curr Biol. 2005 Jul 12;15(13):R483-4. doi: 10.1016/j.cub.2005.06.049.

27. Magnúsdóttir S, Thiele I. Modeling metabolism of the human gut microbiome. Curr Opin Biotechnol. 2018 Jun;51:90-96. doi: 10.1016/j.copbio.2017.12.005. 